\title{
Multiobjective shape optimization of selected coupled problems by means of evolutionary algorithms
}

\author{
A. DŁUGOSZ ${ }^{1 *}$ and T. BURCZYŃSKI ${ }^{1,2}$ \\ ${ }^{1}$ Department of Strength of Materials and Computational Mechanics, Silesian University of Technology \\ 18a. Konarskiego St., 44-100 Gliwice, Poland \\ ${ }^{2}$ Institute of Computer Science, Cracow University of Technology, 24 Warszawska St., 31-155 Cracow, Poland
}

\begin{abstract}
In present paper an improved multi-objective evolutionary algorithm is used for Pareto optimization of selected coupled problems. Coupling of mechanical, electrical and thermal fields is considered. Boundary-value problems of the thermo-elasticity, piezoelectricity and electro-thermo-elasticity are solved by means of finite element method (FEM). Ansys Multiphysics and MSC.Mentat/Marc software are used to solve considered coupled problems. Suitable interfaces between optimization tool and the FEM software are created. Different types of functionals are formulated on the basis of results obtained from the coupled field analysis. Functionals depending on the area or volume of the structure are also proposed. Parametric curves NURBS are used to model some optimized structures. Numerical examples for exemplary three-objective optimization are presented in the paper.
\end{abstract}

Key words: multiobjective optimization, evolutionary algorithms, multiphysics, coupled field problems, finite element method.

\section{Introduction}

Shape optimization of structures is an important phase in engineering design. Real-world problems often have multiple conflicting objectives. It requires the application of efficient multi-objective optimization tools, especially for complex issues. In multi-objective optimization problems, there are several objectives or cost functions to be minimized or maximized simultaneously. Conflicting objectives cause that one objective function improves and the rest deteriorate. Obviously, in these problems there is no single solution which is the best with respect to all objectives. The designer has to chose a solution from a set of solutions which are called optimal in the Pareto sense. For the Pareto optimal solution there exists no other feasible solution which would decrease some objectives (suppose a minimization problem) without causing the simultaneous increase at least one other objective. With this definition of optimality after optimization several tradeoff solutions are obtained (Pareto optimal set). An approach based on the Pareto frontier is considerably faster and more convenient comparing for instance to $\varepsilon$-constraint method or weighting method.

In the present paper multiobjective shape optimization is performed for selected multiphysics tasks. Three different coupled filed problems are considered: thermoelesticity, piezoelectricity and coupling of electrical, thermal and mechanical fields. Boundary-value problems are solved by means of the finite element method (FEM) [1-3]. To solve considered coupled problems FEM Ansys Multiphysics and MSC.Mentat/Marc software packages are used [4, 5]. Functionals are formulated on the basis of results obtained from the coupled field analysis. The boundary element method (BEM) is an adequate technique for solving different optimization tasks also [6]. Shape optimization of thermoelastic structures in the presence of radiation was presented in [7].

Among many different types of multi-objective genetic and evolutionary algorithms [8], Strength Pareto Evolutionary Algorithm [9] and Non-Dominated Sorting Genetic Algorithm [10-12] are the most popular multiobjective optimization tools. Consecutive versions of such algorithms SPEA2 and NSGAII represent the state-of-the-art in evolutionary multi-objective optimization problems and have many practical applications in different engineering disciplines. In this work, in-house implementation of the MultiObjective OPTIMization tool based on evolutionary algorithm (MOOPTIM) is used for optimization [13-16]. Some specific features implemented in NSGAII are applied in MOOPTIM as well.

\section{Formulation of the multiobjective optimization problem}

The process of finding a vector of decision variables that satisfies some restrictions and optimizes the vector of functionals is called multiobjective optimization (MOO). The MOO problem is formulated as follows:

find the vector $\mathbf{x}=\left[x_{1}, x_{2}, \ldots, x_{n}\right]^{T}$ which satisfy the $m$ inequality constrains:

$$
g_{i}(\mathbf{x}) \geq 0 \quad i=1,2, \ldots, m
$$

and the $p$ equality constrains

$$
h_{i}(\mathbf{x})=0 \quad i=1,2, \ldots, p
$$

which minimizes the vector of $k$ objective functions:

$$
f(\mathbf{x})=\left[f_{1}(\mathbf{x}), f_{2}(\mathbf{x}), \ldots, f_{k}(\mathbf{x})\right]^{T},
$$

\footnotetext{
*e-mail: adam.dlugosz@polsl.pl
} 
A. Długosz and T. Burczyński

where $n$ is the number of design variables, $k$ is the number of objective functions.

On each design variable box constraints are imposed

$$
x_{i}^{L} \leq x_{i} \leq x_{i}^{R},
$$

where $x_{i}^{L}$ and $x_{i}^{R}$ are minimum and maximum acceptable values for the variable $x_{i}$ respectively.

Multi-objective optimization deals with multiple conflicting objectives and usually the optimal solution for one of the objectives is not necessarily the optimum for any of the other objectives. For such a case, instead of one optimal solution like in single-objective optimization problem, many solutions are incomparable and optimal. These solutions are called Pareto-optimal ones.

\section{Optimization algorithm}

MultiObjective OPTIMization (MOOPTIM) tool is used for solving multiobjective tasks. As mentioned in Chapter 1 MOOPTIM uses some specific features implemented in NSGAII. Figure 4 shows the flowchart of the MOOPTIM. In the initialization step, besides determining all settings of the algorithm, populations $\mathrm{Q}_{i}$ and $\mathrm{P}_{\mathrm{i}}$ are randomly generated and the fitness functions are evaluated for population $\mathrm{Q}_{\mathrm{i}}$. In the main loop, after evaluation fitness functions for $\mathrm{P}_{\mathrm{i}}$ and checking stop conditions, populations $\mathrm{Q}_{\mathrm{i}}$ and $\mathrm{P}_{\mathrm{i}}$ are combined. The nondominated sorting procedure, proposed by Deb in NSGAII [11] is used for classification of the individuals in population $\mathrm{R}_{\mathrm{i}}$.

In this procedure the population is sorted on the basis of the nondomination. A rank equal to its nondomination level is assigned to each solution. Solutions on front 1 state the best level, solutions on front 2 form the next best level, and so on. This procedure for minimization problem is shown in Fig. 1.

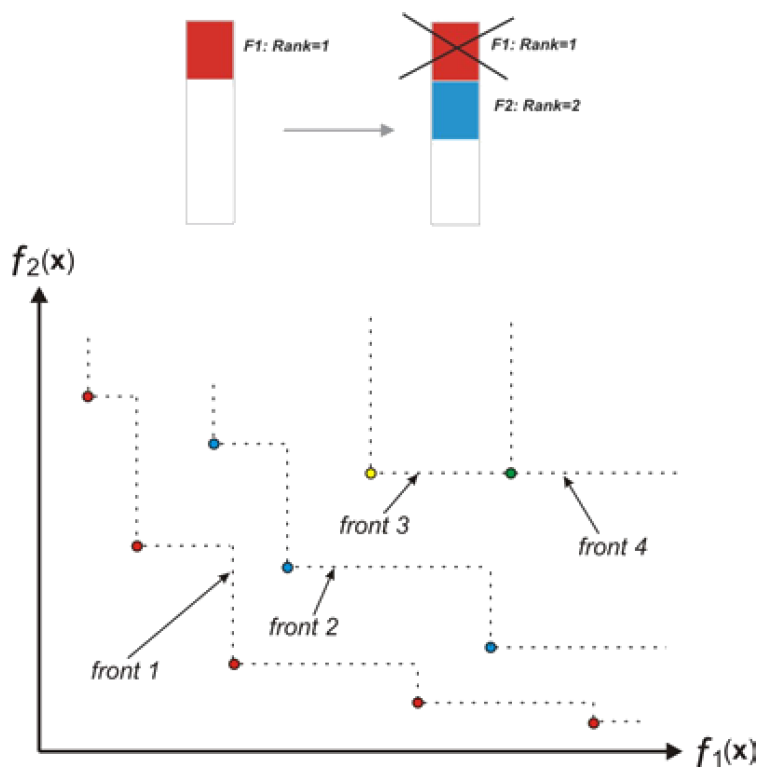

Fig. 1. Classification of the individuals

A crowding coefficient is calculated for each solution [1012]. For calculation of the crowding coefficient the population is sorted according to an objective function value. Solutions with smallest and largest function values (boundary solutions) are assigned as infinite distance value. For all other solutions coefficients are calculated as absolute normalized differences of the function values of two neighboring solutions (Fig. 2).

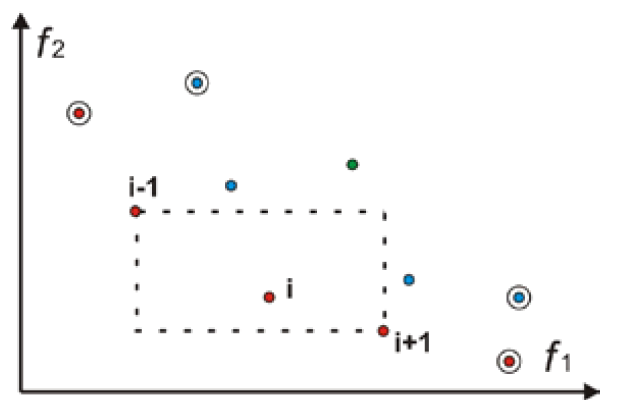

Fig. 2. Calculation of the crowding coefficient

Selection is performed on the set $R_{i}$, which is twice bigger than $P_{i}$. The individuals from the population $R_{i}$ are copied into the population $\mathrm{P}_{\mathrm{i}+1}$ on the basis of the nondomination level and the crowding coefficient (Fig. 3).

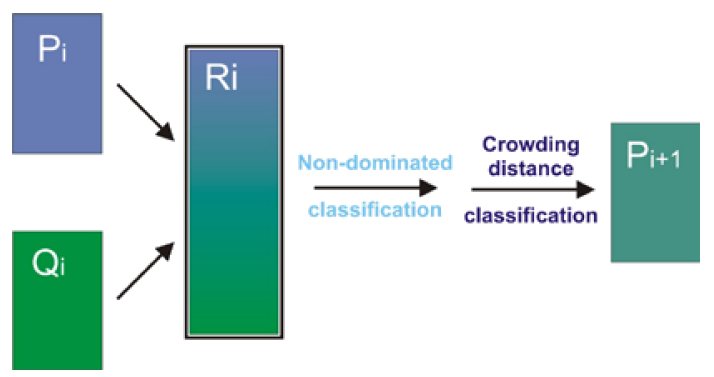

Fig. 3. Scheme of the creation of the next generation

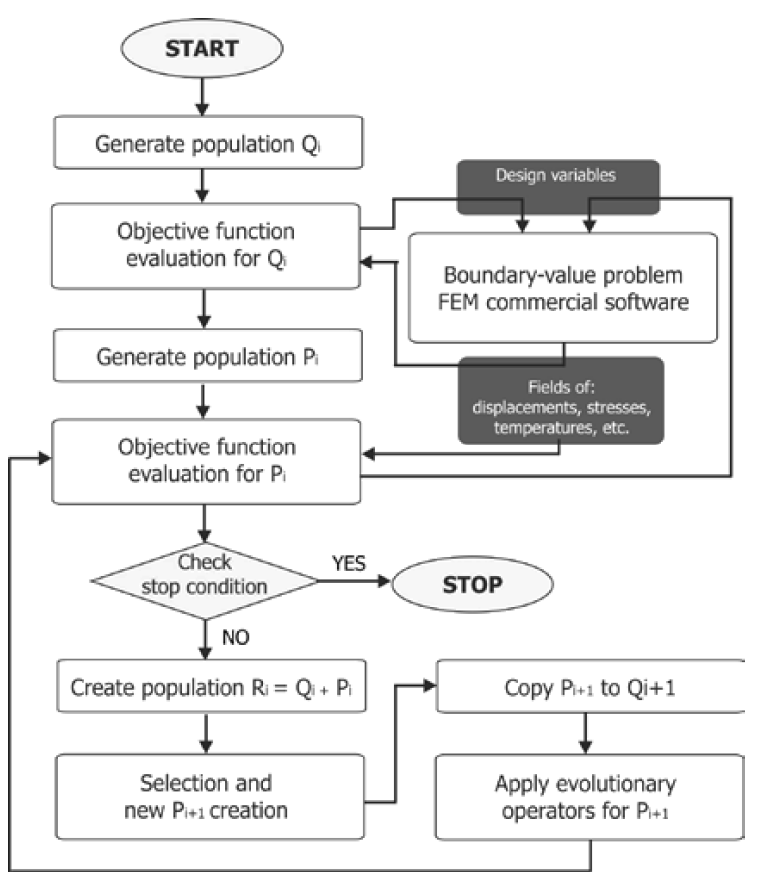

Fig. 4. Flowchart of MOOPTIM 
Multiobjective shape optimization of selected coupled problems...

Individuals from $\mathrm{P}_{\mathrm{i}+1}$ are copied to $\mathrm{Q}_{\mathrm{i}+1}$ and then evolutionary operators change the population $\mathrm{P}_{\mathrm{i}+1}$. Two types of mutation (uniform and Gaussian) and two types of crossover (simple and arithmetic) are used. The algorithm works until stop condition in fulfilled.

Compared to the NSGAII, the proposed implementation has more evolutionary operators. Another difference between these algorithms is related to the formation of parent population - there is no binary tournament selection operator like in NSGA-II, but selection is performed on the basis of nondomination level and crowding coefficient (Fig. 3). The algorithm was tested on several benchmarks (SCH, ZDT1, ZDT2, ZDT3, ZDT4, ZDT6, DTLZ1, DTLZ2, CONSTR, SRN, TNK [11]) and also on some engineering problems. The results obtained using MOOPTIM in most cases are better in comparison with the results obtained by means of NSGA-II. Detailed comparison between MOOPTIM and NSGA-II can be found in [17]. Functionals defining for engineering coupled problems, which is solved by using FEM, are usually strongly multimodal. Ability of finding global solutions by optimization algorithm for such problems is essential.
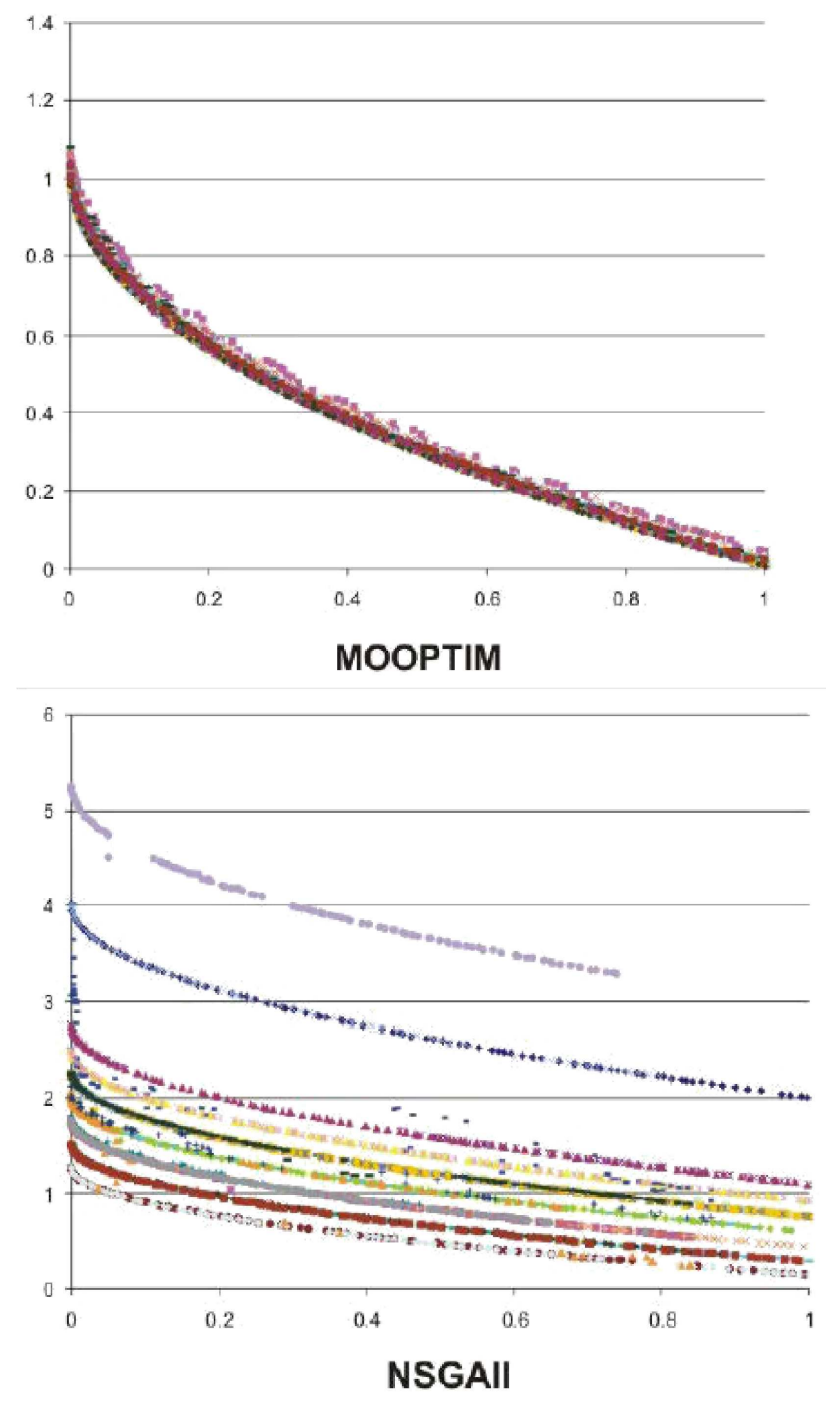

Fig. 5. MOOPTIM-NSGAII comparison on ZDT-4 problem
Figure 5 presents an example of the effectiveness of MOOPTIM algorithm compared to the NSGAII for the ZDT4 problem. The problem has a large number of local Pareto fronts. Computations has been performed for the same number of fitness evaluations. 30 independent runs of both algorithms were performed. MOOPTIM founds a set of Pareto optimal solutions for most cases, whereas NSGA-II very often stuck in local optima.

\section{Formulation of the problem}

In the present work following types of boundary value problems are considered: thermoeleasticity, piezo-electricity and electrical-thermal-mechanical analysis $[18,19]$. These problems are described by the appropriate partial differential equations. The equations with arbitrary geometries and boundary conditions are usually solved by numerical methods. FEM is used to solve boundary-value problems in all cases. Thermoelesticity and electro-thermoelasticity are weakly coupled, what requires solving electrical, thermal and mechanical analysis separately. Coupling is carried out by transferring loads between the considered analysis and using staggered procedures. Matrix equations of electrical, thermal and mechanical problem can be expressed as follows:

$$
\begin{gathered}
\mathbf{K}_{\mathbf{E}} \mathbf{V}=\mathbf{I}, \\
\mathbf{K}_{\mathbf{T}} \mathbf{T}=\mathbf{Q}+\mathbf{Q}_{\mathbf{E}}, \\
\mathbf{K}_{\mathbf{M}} \mathbf{u}=\mathbf{F}+\mathbf{F}_{\mathbf{T}},
\end{gathered}
$$

where $\mathbf{K}_{E}$ is the electrical conductivity matrix, $\mathbf{K}_{\mathbf{T}}$ is the thermal conductivity matrix, $\mathbf{K}_{\mathbf{M}}$ is the stiffness matrix, $\mathbf{Q}_{\mathbf{E}}$ is the heat generation vector due to current flow, $\mathbf{F}_{\mathbf{T}}$ is the force due to thermal strain vector, $\mathbf{V}, \mathbf{T}, \mathbf{u}$ are the nodal vector of voltage, temperature and displacements, respectively, I, $\mathbf{Q}, \mathbf{F}$, are the nodal vector of current, heat fluxes and applied forces, respectively. The thermal and mechanical problems are coupled through thermal strain loads $\mathbf{F}_{\mathbf{T}}$. Coupling between the electrical and thermal problems is done by heat generation due to the electrical flow $\mathbf{Q}_{\mathbf{E}}$.

Piezoelectricity couples electrical and mechanical fields. This problem is solved by means of strong coupling method $[15,20,21]$. It requires the usage of coupled finite elements, which have all mechanical and electric degrees of freedom (displacements and electric potential). Matrix equations of static piezoelectricity can be expressed as follows:

$$
\left[\begin{array}{ll}
\mathbf{K}_{\mathbf{u u}} & \mathbf{K}_{\mathbf{u} \varphi} \\
\mathbf{K}_{\varphi \mathbf{u}} & \mathbf{K}_{\varphi \varphi}
\end{array}\right]\left[\begin{array}{l}
\mathbf{u} \\
\mathbf{\Phi}
\end{array}\right]=\left[\begin{array}{l}
\mathbf{F}_{\mathbf{u}} \\
\boldsymbol{\rho}_{\varphi}
\end{array}\right]
$$

where $\mathbf{K}_{\mathbf{u u}}$ is mechanical stiffness matrix, $\mathbf{K}_{\mathbf{u} \varphi}, \mathbf{K}_{\varphi \mathbf{u}}$ are piezoelectric stiffness matrices, $\mathbf{K}_{\varphi \varphi}$ is dielectric stiffness matrix, $\mathbf{F}_{\mathbf{u}}$ is force vector and $\rho_{\varphi}$ is charge flux vector.

Ansys Multiphysics and MSC.Mentat/Marc are FEM software packages are especially dedicated for solving coupled boundary-value problems [4, 5]. Several coupled problems can be simulated with the use of those software packages. Ansys Multiphysic is used for solving piezoelectricity, whereas MSC.Marc is used for solving thermoelesticity and electrothermo-elasticity. 
A. Długosz and T. Burczyński

Communication between MOOPTIM and FEM packages requires implementation of the proper interfaces. On the basis of chromosome genes, which represent set of design variables, the geometry of the optimized structure is generated. In the next steps the finite element mesh is generated, the boundary conditions are applied and others settings of the analysis are defined. All this steps are performed with the use of own inhouse software and codes written in script languages (Python and APDL) implemented in MSC.Mentat and Ansys preprocesors. After solving coupled analysis, the values of functional are calculated on the basis of output files generated by MSC.Marc and Ansys. In each iteration of the optimization algorithm all this steps are performed for every chromosome. It should be underlined that efficient calculation of this step is critical taking into account the time of the optimization.

\section{Definition of the functionals}

MOOPTIM is applied to the shape optimization of different structures by the minimization or maximization of appropriate functionals.

Different functionals based on the results derived from coupled field analyses are formulated. For the considered problems functionals can be calculated on the basis of nodal results of electrical, thermal and mechanical quantities. Four different functionals are proposed:

- The minimization of the volume of the structure

$$
\min _{\mathbf{x}} f_{1} \stackrel{\text { def }}{=} \int_{\Omega} d \Omega
$$

- The minimization of the maximal value of the equivalent stress

$$
\min _{\mathbf{x}} f_{2} \stackrel{\text { def }}{=} \max \left(\sigma_{e q}\right)
$$

- The maximization of the maximal value of vertical deflection of the structure at node $i$

$$
\max _{\mathbf{x}} f_{3} \stackrel{\text { def }}{=} \max \left(u_{i}\right)
$$

- The minimization of the displacement functional:

$$
\min _{\mathbf{x}} f_{4} \stackrel{\text { def }}{=} \int_{\Omega} \varphi(\mathbf{u}) d \Omega,
$$

where $\varphi(\mathbf{u})=\left[\frac{|\mathbf{u}|}{u_{0}}\right]^{l}, \mathbf{u}$ is the field of displacements, $u_{0}$ is a reference displacement, $l$ is natural number.

\section{Numerical examples}

Example 1. The joint between the block and pipe presented in Fig. 6. is considered in the framework of thermo-elasticity. The cross section of the pipe is parameterized with the use of NURBS curve [22] consisting of 8 control points (Fig. 7).

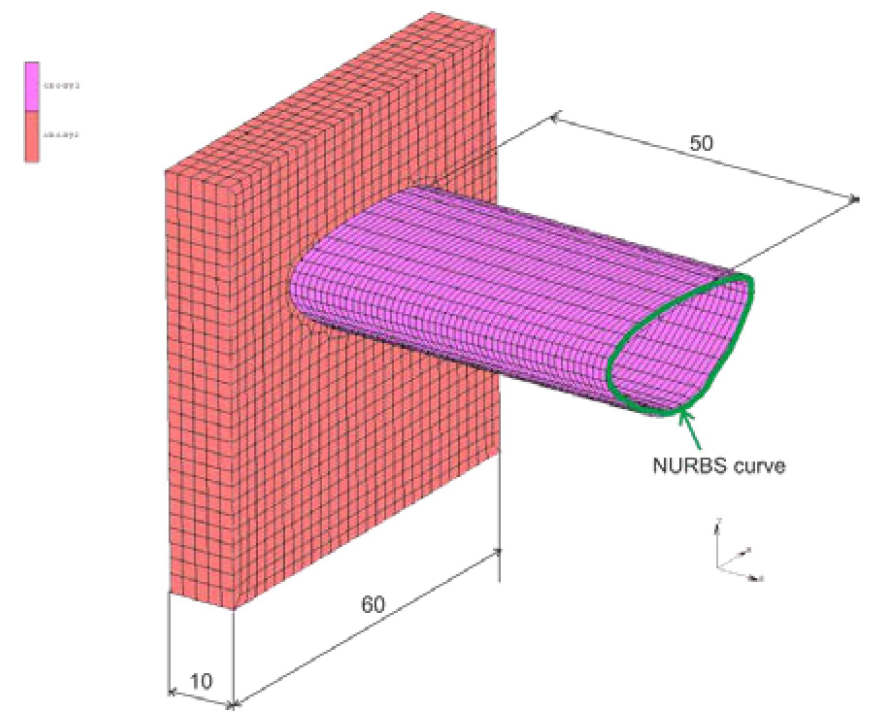

Fig. 6. Joint between block and pipe

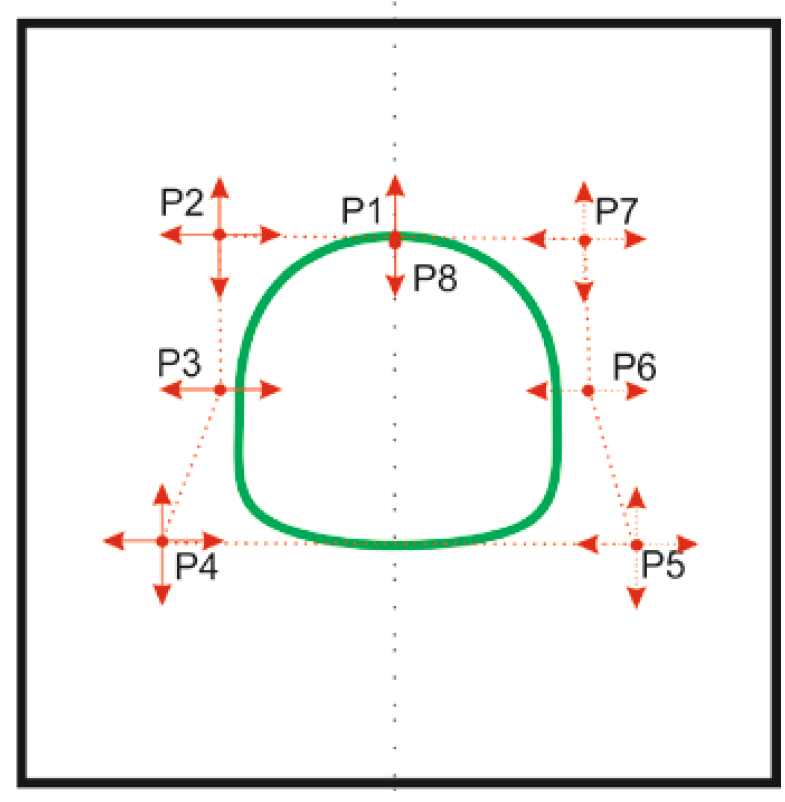

Fig. 7. Parameterization of the cross-section of the pipe

Due to the symmetry and close shape of the curve five design variables are responsible for the control polygon of the NURBS curve (points P2, P3, P4 are symmetrical to P7, P6, $\mathrm{P} 5$ respectively). Moreover the thickness of the pipe is the design variable also. The limitations of the six design variables (Z1-Z6) are collected in Table 1.

Table 1

Limitations of the design variables for example 1

\begin{tabular}{cc}
\hline \hline Design variable & Range $[\mathrm{m}]$ \\
\hline Z1 (horizontal coordinate of $\mathrm{P} 1, \mathrm{P} 2, \mathrm{P} 8)$ & $<0.005 \div 0.025>$ \\
\hline $\mathrm{Z} 2$ (vertical coordinate of $\mathrm{P} 2)$ & $<0.035 \div 0.055>$ \\
\hline $\mathrm{Z} 3$ (horizontal coordinate of $\mathrm{P} 3)$ & $<0.005 \div 0.03>$ \\
\hline Z4 (vertical coordinate of P4) & $<0.005 \div 0.025>$ \\
\hline Z5 (horizontal coordinate of P4) & $<0.005 \div 0.025>$ \\
\hline Z6 (thickness of the pipe) & $<0.0005 \div 0.003>$ \\
\hline
\end{tabular}




\section{Multiobjective shape optimization of selected coupled problems...}

Both the block and the pipe are made of steel. The block consists of solid elements, whereas the pipe is modelled with the use of shell elements. Five surfaces of the block are supported at the end of the pipe. A distributed load $\mathrm{P}_{0} .=600 \mathrm{~N}$ is applied to the end of the pipe. Moreover, a temperature gradient is applied $\left(\mathrm{T} 1=25^{\circ} \mathrm{C}, \mathrm{T} 2=80^{\circ} \mathrm{C}\right)$ to the block and the pipe. Figure 8 shows the thermomechanical boundary conditions for the structure. Between block and the pipe the glue contact and ideal thermal contact are applied.

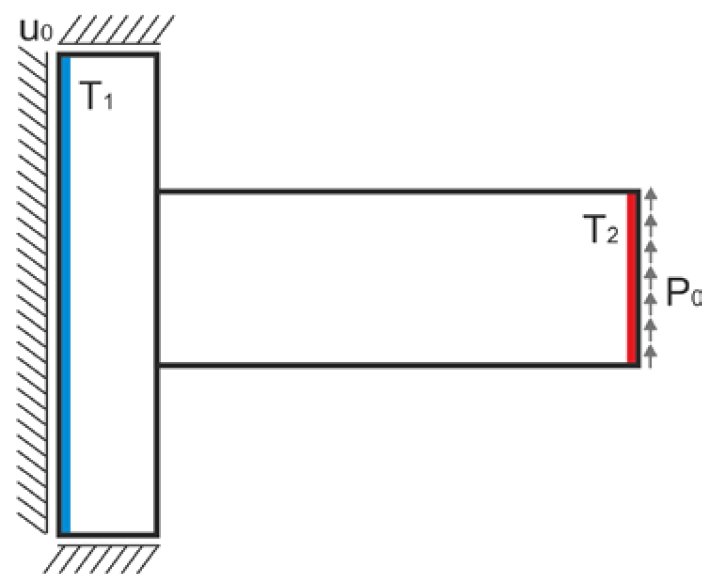

Fig. 8. Boundary condition for the block-pipe joint

The multicriteria optimization task is performed for the functionals (9), (10) and (12). For functional (12) $u_{0}=1$ and $l=1$ are assumed. MOOPTIM tool is run for population size and number of iterations equal to 100 . Probability of uniform mutation is equal to 0.1 , probability of Gaussian mutation is equal to 0.8 , probability of simple and arithmetic crossover is equal to 0.25 .

Figure 9 presents the set of Pareto-optimal solutions, whereas Fig. 10 shows obtained geometry, stress distribution and values of design variables for three selected extreme points on the front.

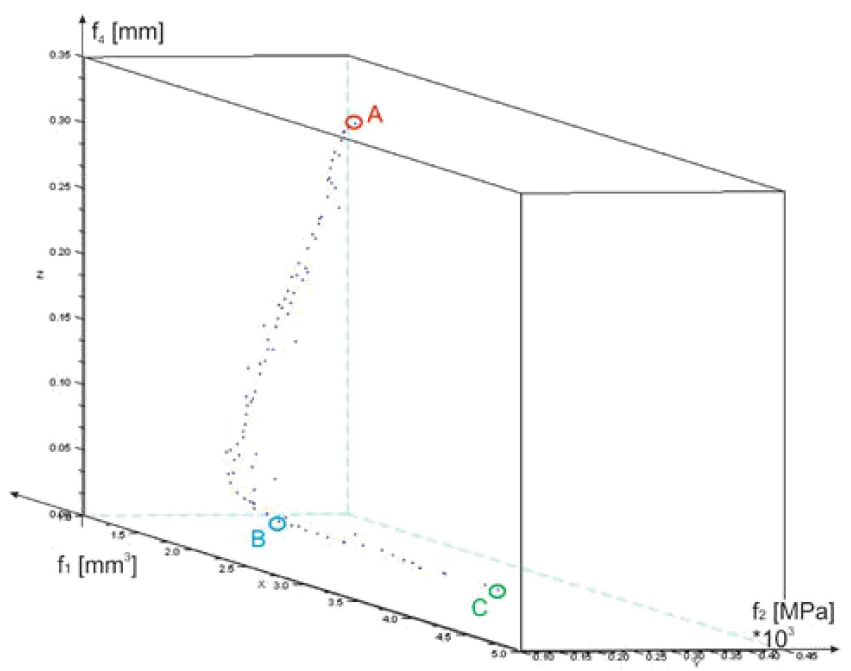

Fig. 9. Set of Pareto solutions for example 1

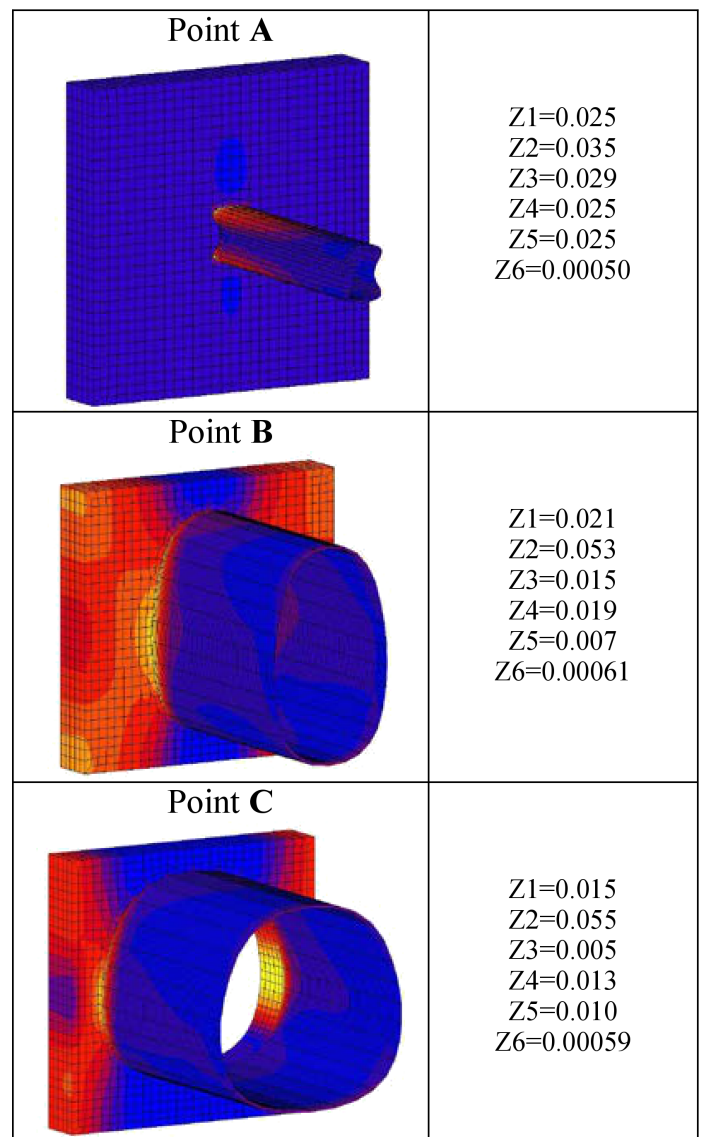

Fig. 10. Example 1: obtained geometry, stress distribution and values of design variables for the points $\mathrm{A}, \mathrm{B}$, and $\mathrm{C}$

For the minimization of the structure volume (9) (Point A) the cross-section of the pipe is similar to I-beam shape. Equivalent stresses for this case are generally caused by bending. For minimization of the equivalent stress (10) and maximization of the stiffness (12) (Points B and C) the cross sections of the pipe have elliptical shapes and thermal stresses overtake stresses caused by bending.

Example 2. The piezoelectric actuator presented in Fig. 11 is considered. The problem is considered as a two dimensional plane stress analysis task. The plate is $100 \mathrm{~mm}$ long, $1 \mathrm{~mm}$ thick and it is made of aluminum. The left side of the plate is fixed. Three electrodes made of the piezoelectric material PZT4 are glued to the plate on the top surface of the plate. A potential difference $1000 \mathrm{~V}$ is applied to the electrodes, which makes the plate bending.

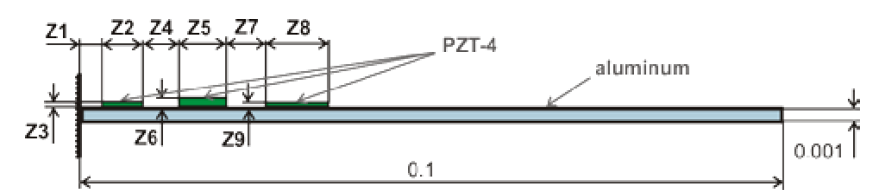

Fig. 11. Model of the piezoelectric actuator

The task is to determine the width, the thickness and locations of the piezoelectric elements. Nine design variables are assumed (Fig. 11). 


\section{A. Długosz and T. Burczyński}

The multiobjective problem is solved taking into account three functional: (9), (10) and (11) simultaneously. In the functional (11) node at the right upper corner of the aluminium beam is considered. Table 1 contains limitations of the design variables and multicriteria algorithm parameters.

Table 2

Limitations of the design variables for actuator

\begin{tabular}{cc}
\hline \hline Design variable & Range $[\mathrm{m}]$ \\
\hline $\mathrm{Z} 1, \mathrm{Z} 4, \mathrm{Z7}$ & $<0.0001 \div 0.01>$ \\
\hline $\mathrm{Z} 2, \mathrm{Z} 5, \mathrm{Z} 8$ & $<0.001 \div 0.1>$ \\
\hline $\mathrm{Z} 3, \mathrm{Z} 6, \mathrm{Z} 9$ & $<0.0005 \div 0.002>$ \\
\hline
\end{tabular}

Figure 12 presents a set of Pareto optimal solutions. Table 2 contains values of design variables for three selected extreme points. Figure 13 contains final shapes, locations of the PZT4 electrodes, stress distribution and values of design variables in this area.

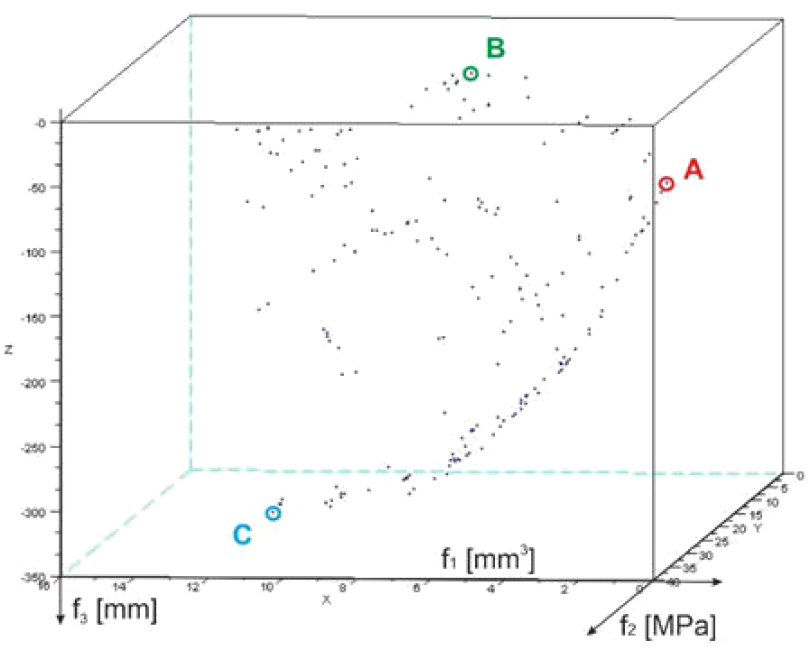

Fig. 12. Set of Pareto solutions for example 2

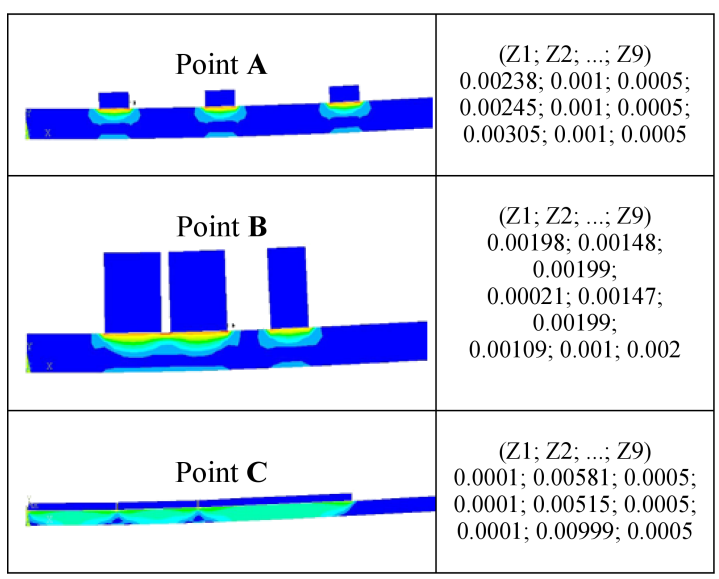

Fig. 13. Obtained geometry of actuator, stress distribution and values of design variables for the points $\mathrm{A}, \mathrm{B}$, and $\mathrm{C}$
For the minimization of the volume (9) MOOPTIM founds minimal possible areas of piezoelectric parts. It is obvious, that Z2, Z5, Z8, Z3, Z6, Z9 should have minimal admissible values, but distances between the electrodes are not intuitive. For the minimization of the stress functional (10) and for maximization of the deflection (11) electrodes have maximal and minimal admissible thicknesses respectively.

Example 3. The model of microelectrothermal actuator is considered (Fig. 14). The actuator is fabricated from polycrystalline silicon which material properties are shown in Table 3 .

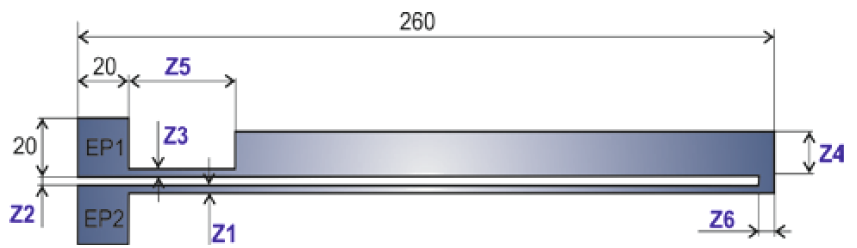

Fig. 14. Model of microelectrothermal actuator

Table 3

Parameters of the polycrystalline silicone

\begin{tabular}{cc}
\hline \hline Parameter & Value \\
\hline Young modulus & $158 \mathrm{e} 3 \mathrm{MPa}$ \\
\hline Poisson ratio & 0.23 \\
\hline Thermal expansion coef. & $3.0 \mathrm{e}-61 / \mathrm{K}$ \\
\hline Thermal conductivity & $140 \mathrm{e} 8 \mathrm{pW} / \mu \mathrm{mK}$ \\
\hline Resistivity & $3.3 \mathrm{e}-11 \mathrm{~T} \Omega \mu \mathrm{m}$ \\
\hline
\end{tabular}

The deflection of the actuator occurs when the electrical potential difference is applied across two electrical pads. It is possible due to material properties - high electrical resistivity and different thermal expansion between thin and wide arms. The device is subjected to the electrical, thermal and mechanical boundary conditions. The length of the actuator is equal to 260 microns, while electrical pads are $20 \times 20$ microns wide. The multiobjective problem concerns determining the specified dimensions of the actuator shape, which minimize or maximize functionals (9), (10) and (11). In the functional (11) node at the right upper corner is considered. Six design variables are assumed (Fig. 7). Table 4 contains limitations of the design variables. Parameters of the MOOPTIM algorithm are identical as in Example 1. Figure 15 presents a set of Pareto optimal solutions, whereas Fig. 16 contains final shapes, stress distribution and values of design variables for points A, B and C. The results obtained for functionals (9) and (10) are similar, whereas the optimal shape obtained for functional (11) reminds shapes very often applied in practice (see examples presented on the web site of Institute of Micromachine and Microfabrication Research and Simon Fraser University [23, 24]). 
Table 4

Limitations of the design variables

\begin{tabular}{cc}
\hline \hline Design variable & Range $[\mu \mathrm{m}]$ \\
\hline $\mathrm{Z} 1, \mathrm{Z} 2, \mathrm{Z} 3$ & $i 1 \div 3 i$ \\
\hline $\mathrm{Z} 4$ & $i 12 \div 18_{i}$ \\
\hline $\mathrm{Z} 5$ & $i^{30} \div 100_{i}$ \\
\hline $\mathrm{Z} 6$ & $i^{2} \div 8 i$ \\
\hline
\end{tabular}

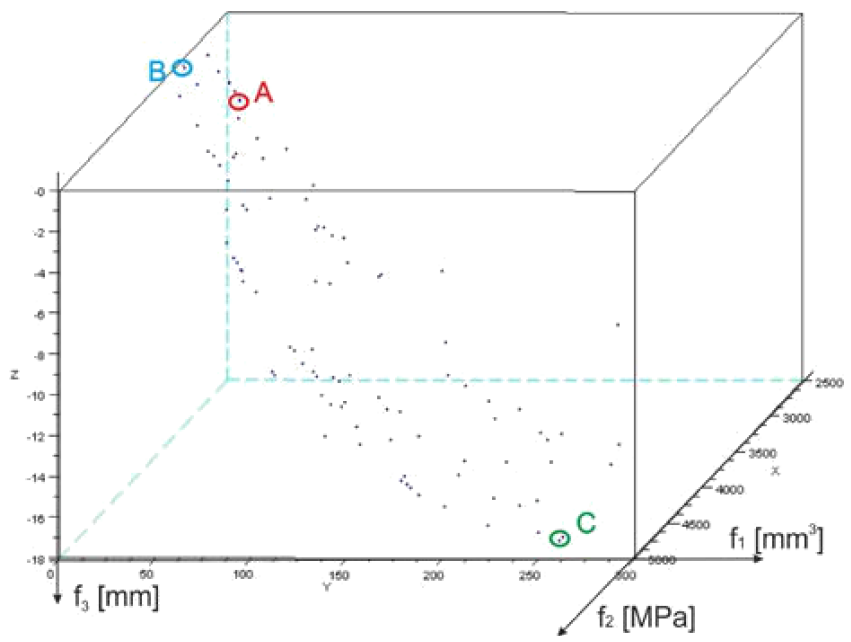

Fig. 15. Set of Pareto solutions

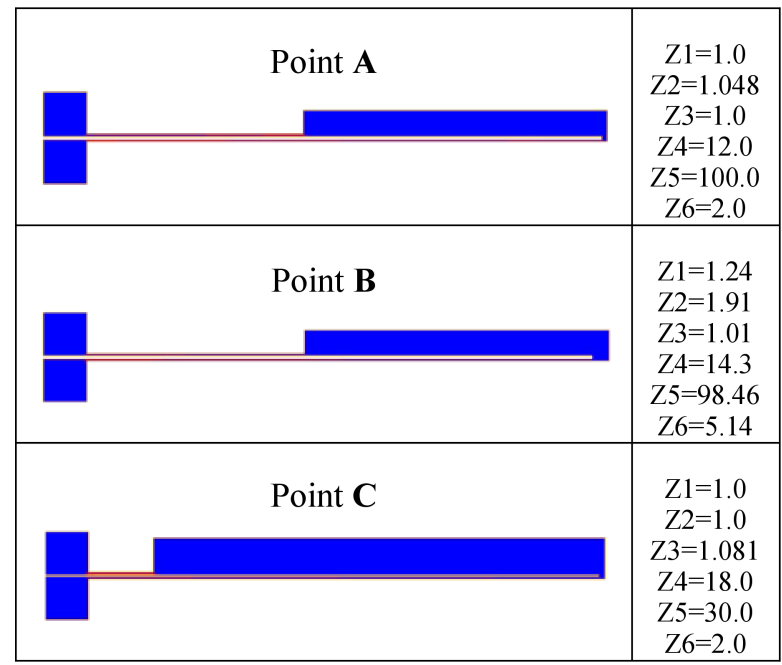

Fig. 16. Obtained geometry, stress distribution and values of design variables for the points $\mathrm{A}, \mathrm{B}$, and $\mathrm{C}$

\section{Final remarks}

The designing of the real structures, especially for multiphysics problems, when different criteria are taken into account, belongs to difficult optimization tasks. Intuitive solutions can be found only for some cases (simple geometry, simple boundary conditions, low number of design variables, etc.). The application of classical methods based on gradient algorithms may be also unsuccessful due to the multimodality of functionals for real problems.

In the present work the MOOPTIM algorithm has been used for multiobjective shape optimization of different structures. The algorithm based on evolutionary algorithms be- longs to global optimization methods. The efficiency of such optimization tool was tested on several benchmark problems. In most cases MOOPTIM gives better results comparing to the NSGA-II algorithm.

The direct problems concern coupling between mechanical, thermal and electrical fields. For solving direct coupled problems MSC.Mentat/Marc and Ansys Multiphysics are applied. Interfaces between MOOPTIM and FEM software's are prepared with the use of in-house software and codes written in script languages.

The problems were solved for different criteria based on thermal, mechanical and other quantities. Another type of functionals can be formulated. Application of parametric curves in preparation of the model can reduce the number of design variables.

The application of the FEM software requires evaluation in several steps for each single solution (the modification of the geometry, creating finite element mesh, etc.). It can be very-time consuming task, especially for more complicated geometries. Moreover the solution of the coupled problems is more time-consuming in comparison to the single-field problems. For the numerical models considered in the paper the time needed for solving one multiciriteria optimization task may takes several hours.

In order to reduce the time of the computation the future tasks are: application of approximate surrogate model and parallelization of the fitness function evaluations.

Acknowledgements. The research was financed by the Polish science budget resources within the years 2008-2011 as a research project.

\section{REFERENCES}

[1] G. Beer, "Finite element, boundary element and coupled analysis of unbounded problems in elastostastics", Int. Meth. Eng. 19, 567-580 (1983).

[2] M. Kleiber, Handbook of Computational Solid Mechanics, Springer, Berlin, 1998.

[3] O.C. Zienkiewicz and R.L. Taylor, The Finite Element Method, Butterworth Heinemann, Oxford, 2000.

[4] Ansys Multiphysics Documentation, Ansys Co, 2010.

[5] MSC.MARC, Theory and User Information, Vol. A-D, MSC Software Corporation, 2010.

[6] T. Burczyński and A. Długosz, "Evolutionary optimization in thermoelastic problems using the boundary element method", Computational Mechanics 28 (3-4), 317-324 (2002).

[7] R. Białecki, T. Burczyński, A. Długosz, W. Kuś, and Z. Ostrowski, "Evolutionary shape optimization of thermolastic bodies exchanging heat by convection and radiation", Computer Methods in Appl. Mechanics and Eng. 194, 1839-1859 (2005).

[8] Z. Michalewicz, Genetic Algorithms + Data Structures $=$ Evolutionary Programs, WNT, Warszawa, 1996, (in Polish).

[9] E. Zitzler, M. Laumanns, and L. Thiele, "SPEA2: improving the strength pareto evolutionary algorithm", TIK-Report 103, CD-ROM (2001).

[10] K. Deb, "Multi-objective genetic algorithms: problem difficulties and construction of test problems", Evolutionary Computation 7 (3), 205-230 (1999). 
A. Długosz and T. Burczyński

[11] K. Deb, A. Pratap, S. Agarwal, and T. Meyarivan, "A fast and elitist multi-objective genetic algorithm: NSGA-II", IEEE Trans. on Evolutionary Computation 6 (2), 181-197 (2002).

[12] K. Deb, "Evolutionary multi-objective optimization without additional parameters, studies in computational intelligence", Evolutionary Comp. 54, 241-257 (2007).

[13] A. Długosz and T. Burczyński, "Integration of multiobjective evolutionary algorithms and CAE systems in shape optimization of selected coupled problems", Coupled Problems IV Comp. Methods for Coupled Problems in Science and Eng. 1, CD-ROM (2011).

[14] A. Długosz and T. Burczyński, "Shape optimization of electrothermal-mechanical systems by using multiobjective evolutionary algorithms", Evolutionary and Deterministic Methods for Design, Optimization and Control. Applications to Industrial and Societal Problems CIMNE 1, 162-169 (2011).

[15] G. Dziatkiewicz, A. Długosz, and T. Burczyński, "Application of multi-objective evolutionary algorithms in optimization of piezoelectric models", IV Eur. Conf. on Comp. Mechanics (ECCM IV) 1, CD-ROM (2010).

[16] W. Kuś, A. Długosz, and T. Burczyński, "OPTIM - library of bioinspired optimization algorithms in engineering applications", Computer Methods in Materials Science 11 (1), 9-15 (2011).

[17] A. Długosz, "Multiobjective evolutionary optimization of MEMS structures", Computer Assisted Mechanics and Eng. Sci. 17 (1), 41-50 (2010).

[18] N. Maluf and K. Williams, An Introduction to Microelectromechanical Systems Engineering", Artech House Publishers, London, 2004.

[19] G.A.Maugin, R. Drouot, and F.Sidoroff, Continuum Mechanics, Cluwer Academic Publisher, London, 2002.

[20] G. Helke and K. Lubitz, "Piezoelectric PZT ceramics", Springer Series in Materials Science 114, 89-130 (2008).

[21] A. Poteralski, M. Szczepanik, G. Dziatkiewicz, W. Kuś, and T. Burczyński, "Immune identification of piezoelectric material constants using BEM", Inverse Problems in Science and Engineering 19 (1), 103-116 (2011).

[22] L.A. Piegl and W. Tiller, The NURBS Book, Springer, Berlin, 1996.

[23] http://www.sfu.ca/immr/

[24] http://www.sfu.ca/adm/heatuator.html 